\title{
MILLIMETER WAVE SCATTERING MODEL FOR A LEAF
}

\author{
K. Sarabandi, F.T. Ulaby, and T.B.A. Senior \\ Radiation Laboratory, Electrical Engineering and Computer Science Department, \\ University of Michigan, Ann Arbor
}

MARCH 1989 


\title{
MILLIMETER WAVE SCATTERING MODEL FOR A LEAF
}

\author{
K. Sarabandi, F.T. Ulaby, and T.B.A. Senior \\ Radiation Laboratory, Electrical Engineering and Computer Science Department, \\ University of Michigan, Ann Arbor
}

\begin{abstract}
At millimeter wave frequencies a typical leaf is a significant fraction of a wavelength in thickness, and its nonuniform dielectric profile now affects the scattering. To provide a simple and efficient method for predicting the scattering, two types of physical optics approximations are examined. The first approximates the volume polarization current by the current which would exist in an infinite dielectric slab with the same profile, while the second (and simpler) one employs the surface current which, on the infinite slab, produces the known reflected field. It is shown that the first method is superior, and provided the actual dielectric profile is used, it predicts the scattered field to an accuracy which is adequate for most practical purposes.
\end{abstract}




\section{Introduction}

Leaves are a key feature of any vegetation canopy, and in order to model the scattering from vegetation-covered land, it is necessary to develop an efficient and effective technique for predicting the scattering from a single leaf. At microwave frequencies where a typical leaf is electrically thin with lateral dimensions at least comparable to the free space wavelength $\lambda_{0}$, several methods have been proposed [e.g. Le Vine et al 1985, Willis et al, 1988] all based on the physical optics approximation applied to a uniform dielectric slab. In particular, if the leaf thickness is no more than about $\lambda_{0} / 50$, physical optics in conjunction with a resistive sheet model predicts the scattering at most angles of incidence [Senior et al, 1987] and can also handle curved leaves [Sarabandi et al, 1988].

On the other hand, at millimeter wavelengths the thickness can be a significant fraction of a wavelength, and it is also necessary to take into account the internal structure of a leaf. At least two different types of cell can be distinguished, and their differing water content affects the dielectric constant, leading to a nonuniform dielectric profile. To compute the scattering at these higher frequencies, two different physical optics approximations are examined. The first of these employs the polarization current which would exist in an infinite slab consisting of one, two or more layers simulating the dielectric profile of the leaf, and this is referred to as the volume integral physical optics (VIPO) approximation. When there are many layers, a convenient method of implementation is described in the Appendix. The second (and simpler) approach postulates a surface current which, for an infinite 
slab, produces a plane wave identical to the reflected field, and this is the surface current physical optics (SCPO) approximation.

For an electrically thin leaf or plate, the two approximations are indistinguishable, but as the thickness (or frequency) increases, the predicted scattering differs in most directions, and by comparison with the results of a moment method solution of the volume integral equation, it is shown that VIPO is superior. In addition, for a two layer material, it is no longer adequate to treat the plate as homogeneous one having an average dielectric constant. Provided the actual dielectric profile of a leaf is simulated, it appears that VIPO can predict the scattering behavior of a leaf to an accuracy that is sufficient for most practical purposes at millimeter wavelengths.

\section{Structure of a Leaf}

The structure of a typical vegetation leaf is shown in Fig.1. The type and number density of cells may vary as a function of depth into the leaf which, in turn, results in a nonuniform dielectric profile. The effect of this nonuniformity becomes observable at higher frequencies where the thickness of the leaf is comparable to the wavelength.

Leaves contain two types of photosynthetic cells: palisade parenchyma, consisting of column-shaped cells in which most photosynthesis takes place, and spongy parenchyma, which consist of irregularly shaped cells with large spaces between them. Because a large part of the vegetation material is water, its dielectric con- 
stant is strongly influenced by the dielectric constant of water and the water content. For most leaves, the water content is higher in its upper layer (palisade region) than in the under surface (spongy region). The sensitivity of the dielectric constant to water content is much greater in the lower part of the millimeter wave spectrum than in the upper, but this is more than counterbalanced by the thickness to wavelength ratio. The net result is that the sensitivity to dielectric variations is greater at the higher frequencies.

To examine the effect of the nonuniform dielectric profile on the scattering properties of the leaf at millimeter wavelengths, we computed the normal incidence reflection coefficient $\Gamma_{0}$ of a two-layer dielectric slab and compared it with the reflection coefficient of a uniform dielectric slab whose dielectric constant is the average. The computation was performed for a leaf thickness of $0.5 \mathrm{~mm}$, and the water content ratio of the two layer was chosen to be 4 to 1 , representing a marked variation between the upper and lower surfaces of the leaf. From the data in Table 1 , it is seen that when the two-layer slab is approximated by a uniform slab the

\begin{tabular}{|c||c|c|c||c|c|}
\hline$f(\mathrm{GHz})$ & $\epsilon_{1}$ & $\epsilon_{2}$ & $\epsilon_{(\text {avg })}=\frac{\epsilon_{1}+\epsilon_{2}}{2}$ & $\Gamma_{0}$ & $\Gamma_{0(a v g)}$ \\
\hline 35 & $20+\mathrm{i} 21$ & $6+\mathrm{i} 3$ & $13+\mathrm{i} 12$ & $0.74 \angle 6$ & $0.78 \angle-0.16$ \\
\hline 94 & $6+\mathrm{i} 5$ & $2+\mathrm{i} 1$ & $4+\mathrm{i} 3$ & $0.59 \angle 12$ & $0.48 \angle 27$ \\
\hline 140 & $5+\mathrm{i} 4$ & $2+\mathrm{i} 1$ & $3.5+\mathrm{i} 2.5$ & $0.50 \angle 20$ & $0.34 \angle 26.1$ \\
\hline
\end{tabular}

Table 1: Voltage reflection coefficient for a two-layer and average dielectric slab error in the reflection coefficient increases with increasing frequency, and is as large 
as $4 \mathrm{~dB}$ at $140 \mathrm{GHz}$.

\section{Physical Optics Approximations}

At microwave frequencies where a typical leaf is no more than about $\lambda_{0} / 50$ in thickness with lateral dimensions comparable to or larger than the wavelength, the scattering properties can be accurately predicted using the physical optics approximation applied to a resistive sheet model of a leaf [Sarabandi et al, 1988]. In effect, the leaf is modeled as an infinitesimally thin layer, but as the frequency increases, it is necessary to take the leaf thickness in to account. There are now two types of physical optics approximation that can be employed. The standard one is the surface current (SCPO) approach in which an infinite dielectric slab is replaced by an equivalent sheet current that produces a plane wave identical to the reflected wave of the slab. This current is then used as an approximation to the equivalent surface current over the upper surface of a finite dielectric plate. Alternatively, the induced (volume) polarization current in the plate can be approximated by the current in the infinite dielectric slab, and we shall refer to this as the volume integral physical optics (VIPO) method. It is more accurate than the SCPO method, although the latter is more convenient to use for evaluating the scattered field.

To illustrate the two procedures, consider a dielectric plate consisting of a homogeneous dielectric of thickness $d_{1}$ and relative permittivity $\epsilon_{1}$ atop a second material of thickness $d_{2}-d_{1}$ and relative permittivity $\epsilon_{2}$. The plate occupies the region $-\frac{a}{2} \leq y \leq \frac{a}{2},-\frac{b}{2} \leq y \leq \frac{b}{2}$, and $-d_{2} \leq z \leq 0$ as shown in Fig. 2, and is 
illuminated by an E-polarized plane wave whose electric vector is

$$
\mathbf{E}^{i}=\hat{\mathbf{y}} e^{i k_{0}\left(x \sin \theta_{0}-z \cos \theta_{0}\right)}
$$

where $k_{0}$ is the propagation constant in the free space medium above and below the plate. When the plate is treated as an infinitely extended slab, the electric field can be written as

$$
\begin{array}{ll}
E_{y}=\left(e^{-i k_{0 z} z}+\Gamma e^{i k_{0} z}\right) e^{i k_{0} \sin \theta_{0} x} & (0 \leq z) \\
E_{y}=\left(B_{1} e^{-i k_{1 z} z}+A_{1} e^{i k_{1 z} z}\right) e^{i k_{0} \sin \theta_{0} x} & \left(-d_{1} \leq z \leq 0\right) \\
E_{y}=\left(B_{2} e^{-i k_{2 z} z}+A_{2} e^{i k_{2 z} z}\right) e^{i k_{0} \sin \theta_{0} x} & \left(-d_{2} \leq z \leq-d_{1}\right) \\
E_{y}=B_{3} e^{-i k_{0} z} e^{i k_{0} \sin \theta_{0} x} & \left(z \leq-d_{2}\right)
\end{array}
$$

where

$$
k_{0 z}=k_{0} \cos \theta_{0}, \quad k_{j z}=k_{0} \sqrt{\epsilon_{j}-\sin ^{2} \theta_{0}}
$$

for $j=1,2$. If $R_{1}$ and $R_{2}$ are the reflection coefficients at the upper and lower surfaces where

$$
R_{1}=\frac{k_{0 z}-k_{1 z}}{k_{0 z}+k_{1 z}}, \quad R_{2}=\frac{k_{0 z}-k_{2 z}}{k_{0 z}+k_{2 z}}
$$

and

$$
C_{ \pm}=1 \pm \frac{k_{2 z}}{k_{1 z}}\left\{1+R_{2} e^{2 i k_{2 z}\left(d_{2}-d_{1}\right)}\right\}\left\{1-R_{2} e^{2 i k_{2 z}\left(d_{2}-d_{1}\right)}\right\}^{-1},
$$

application of the boundary condition at the three interfaces gives

$$
\begin{aligned}
& B_{1}=\frac{C_{+}\left(1+R_{1}\right)}{C_{+}+C_{-} R_{1} e^{2 i k_{1} d_{1}}}, \quad A_{1}=\frac{C_{-}}{C_{+}} e^{2 i k_{1 z} d_{1}} B_{1} \\
& B_{2}=\frac{e^{i\left(k_{1 z}-k_{2 z}\right) d_{1}}}{1-R_{2} e^{2 i k_{2 z}\left(d_{2}-d_{1}\right)}} \cdot \frac{2}{C_{+}} B_{1}, \quad A_{2}=-R_{2} e^{2 i k_{2 z} d_{2}} B_{2} \\
& B_{3}=e^{i\left(k_{2 z}-k_{0 z}\right) d_{2}}\left(1-R_{2}\right) B_{2}
\end{aligned}
$$


and

$$
\Gamma=\frac{C_{+} R_{1}+C_{-} e^{2 i k_{1 z} d_{1}}}{C_{+}+C_{-} R_{1} e^{2 i k_{1 z} d_{1}}} .
$$

The corresponding results for a single layer of thickness $d_{1}$ and relative dielectric constant $\epsilon_{1}$ can be obtained by putting $d_{2}=d_{1}$ and $k_{2 z}=k_{1 z}$, implying $B_{2}=B_{1}$ and $A_{2}=A_{1}$.

Given a volume distribution of electric current $\bar{J}$ in free space, the corresponding Hertz vector is

$$
\Pi(\bar{r})=\frac{i Z_{0}}{4 \pi k_{0}} \int_{V} \mathbf{J}\left(\bar{r}^{\prime}\right) \frac{e^{i k_{0}\left|\bar{r}-\bar{r}^{\prime}\right|}}{\left|\bar{r}-\bar{r}^{\prime}\right|} \mathrm{d} v,
$$

where $Z_{0}\left(=1 / Y_{0}\right)$ is the free space impedance, and the resulting field is

$$
\begin{aligned}
\mathbf{E}(\bar{r}) & =\nabla \times \nabla \times \Pi(\bar{r}), \\
\mathbf{H}(\bar{r}) & =-i k_{0} Y_{0} \nabla \times \Pi(\bar{r}) .
\end{aligned}
$$

In the far zone of the current distribution

$$
\Pi(\bar{r}) \approx \frac{e^{i k_{0} r}}{k_{0} r} \frac{i Z_{0}}{4 \pi k_{0}} \int_{V} \mathbf{J}\left(\bar{r}^{\prime}\right) e^{-i k_{0} \hat{r} \cdot \bar{r}^{\prime}} \mathrm{d} v
$$

and

$$
\mathrm{E}(\bar{r}) \approx-k_{0}^{2} \hat{\mathbf{r}} \times \hat{\mathbf{r}} \times \Pi(\bar{r}) .
$$

In the dielectric slab the volume current $\bar{J}$ is the polarization current

$$
\mathbf{J}=-i k_{0} Y_{0}\left(\epsilon_{j}-1\right) E_{y} \hat{\mathbf{y}}
$$

where $E_{y}$ has the value appropriate to each layer $(j=1,2)$, and when this is inserted into (6) and the integration carried out over the volume occupied by 
the plate, we obtain the VIPO approximation. For scattering in the direction $\theta_{s}$ indicated in Fig. 2 the expression for the Hertz vector is

$$
\Pi^{V I P O}=\hat{\mathrm{y}} \frac{e^{i k_{0} r}}{k_{0} r} \frac{k_{0} a b}{4 \pi} \frac{\sin X}{X} F
$$

where

$$
\begin{aligned}
& F=\left(\epsilon_{1}-1\right)\left\{\frac{1-e^{-i\left(k_{1 z}-k_{0} \cos \theta_{s}\right) d_{1}}}{i\left(k_{1 z}-k_{0} \cos \theta_{s}\right)} A_{1}-\frac{1-e^{-i\left(k_{1 z}+k_{0} \cos \theta_{s}\right) d_{1}}}{i\left(k_{1 z}+k_{0} \cos \theta_{s}\right)} B_{1}\right\}
\end{aligned}
$$

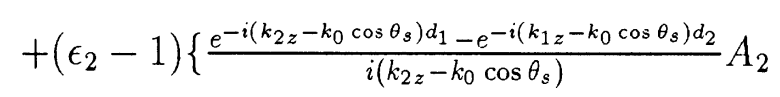

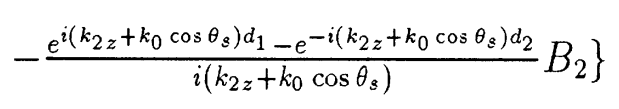

and

$$
X=\frac{k_{0} a}{2}\left(\sin \theta_{s}+\sin \theta_{0}\right)
$$

The far zone scattered field can then be obtained from (7) and written as

$$
\mathrm{E}^{s}=\frac{e^{i k_{0} r}}{k_{0} r} \mathbf{S}_{E}\left(\theta_{s}, \theta_{0}\right)
$$

where $\mathbf{S}_{E}\left(\theta_{s}, \theta_{0}\right)$ is the far field amplitude, and for the VIPO approximation the result is

$$
\mathbf{S}_{E}^{V I P O}\left(\theta_{s}, \theta_{0}\right)=\hat{\mathbf{y}} \frac{k_{0}^{3} a b}{4 \pi} \frac{\sin X}{X} F .
$$

In terms of the far field amplitude, the bistatic scattering cross section is

$$
\sigma\left(\theta_{S}, \theta_{0}\right)=\frac{\lambda_{0}^{2}}{\pi}\left|\mathbf{S}\left(\theta_{s}, \theta_{0}\right)\right|^{2} .
$$

The more conventional SCPO approximation can be obtained by noting that the electric current sheet

$$
\mathbf{J}=-2 Y_{0} \cos \theta_{0} \Gamma e^{i k_{0} \sin \theta_{0} x} \delta(z) \hat{\mathbf{y}}
$$


produces a plane wave identical to the field reflected from the dielectric slab. As evident from the impulse function $\delta(z)$ in (16), the current is located at the upper surface of the slab, and when (16) is inserted into (6) we find

$$
\Pi^{S C P O} \approx \hat{\mathrm{y}} \frac{e^{i k_{0} r}}{k_{0} r} \cdot \frac{-i}{2 \pi} \cos \theta_{0} \Gamma a b \frac{\sin X}{X},
$$

and the far field amplitude is then

$$
\mathrm{S}_{E}^{S C P O}\left(\theta_{s}, \theta_{0}\right)=\hat{\mathbf{y}} \frac{-i k_{0}^{2}}{2 \pi} \cos \theta_{0} \Gamma a b \frac{\sin X}{X} .
$$

In the specular $\left(\theta_{s}=-\theta_{0}\right)$ and backscattering $\left(\theta_{s}=\theta_{0}\right)$ directions it can be verified that (14) and (18) are identical, but in the other directions the two approximations differ.

In the case of $\mathrm{H}$ polarization for which

$$
\mathbf{H}^{i}=\hat{\mathbf{y}} e^{i k_{0}\left(x \sin \theta_{0}-z \cos \theta_{0}\right)}
$$

the analysis is similar. With $H_{y}$ represented as shown in (2), the various coefficients (now indicated by primes) differ from those for $\mathrm{E}$ polarization in having $k_{1 z}$ replaced by $k_{1 z} / \epsilon_{1}$ and $k_{2 z}$ replaced by $k_{2 z} / \epsilon_{2}$ everywhere except in the exponents. The induced polarization current then has two components and is given by

$$
\mathbf{J}=-i k_{0} Z_{0}\left(\epsilon_{j}-1\right)\left(E_{x} \hat{\mathbf{x}}+E_{z} \hat{\mathbf{z}}\right) \text {. }
$$

where $E_{x}=\left(i k_{0} \epsilon_{j}\right)^{-1} Z_{0} \partial H_{y} / \partial x$ and $E_{z}=-\left(i k_{0} \epsilon_{j}\right)^{-1} Z_{0} \partial H_{y} / \partial x$ have the values appropriate to each layer $(j=1,2)$. The Hertz vector can be computed using $(6)$, and for the scattered field $\mathbf{H}^{s}$, the far field amplitude is found to be

$$
\mathbf{S}_{H}^{V I P O}\left(\theta_{s}, \theta_{0}\right)=\hat{\mathrm{y}} \frac{k_{0}^{3} a b}{4 \pi} \frac{\sin X}{X}\left(\cos \theta_{s} F_{1}^{\prime}-\sin \theta_{s} F_{2}^{\prime}\right) .
$$


where

$$
\begin{aligned}
& F_{1}^{\prime}=\left\{\frac{k_{1 z}\left(\epsilon_{1}-1\right)}{k_{0} \epsilon_{1}}\left[\frac{1-e^{-i\left(k_{1 z}-k_{0} \cos \theta_{s}\right) d_{1}}}{i\left(k_{1 z}-k_{0} \cos \theta_{s}\right)} A_{1}^{\prime}+\frac{1-e^{i\left(k_{1 z}+k_{0} \cos \theta_{s}\right) d_{1}}}{i\left(k_{1 z}+k_{0} \cos \theta_{s}\right)} B_{1}^{\prime}\right]\right. \\
& +\frac{k_{2 z}\left(\epsilon_{2}-1\right)}{k_{0} \epsilon_{2}}\left[\frac{e^{-i\left(k_{2 z}-k_{0} \cos \theta_{s}\right) d_{1}}-e^{-i\left(k_{1 z}-k_{0} \cos \theta_{s}\right) d_{2}}}{i\left(k_{2 z}-k_{0} \cos \theta_{s}\right)} A_{2}^{\prime}\right.
\end{aligned}
$$

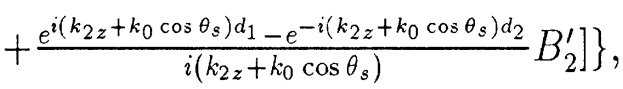

and

$$
\begin{aligned}
& F_{2}^{\prime}=\sin \theta_{0}\left\{\frac{\left(\epsilon_{1}-1\right)}{\epsilon_{1}}\left[\frac{1-e^{-i\left(k_{1 z}-k_{0} \cos \theta_{s}\right) d_{1}}}{i\left(k_{1 z}-k_{0} \cos \theta_{s}\right)} A_{1}^{\prime}-\frac{1-e^{i\left(k_{1 z}+k_{0} \cos \theta_{s}\right) d_{1}}}{i\left(k_{1 z}+k_{0} \cos \theta_{s}\right)} B_{1}^{\prime}\right]\right. \\
& +\frac{\left(\epsilon_{2}-1\right)}{\epsilon_{2}}\left[\frac{e^{-i\left(k_{2 z}-k_{0} \cos \theta_{s}\right) d_{1}}-e^{-i\left(k_{1 z}-k_{0} \cos \theta_{s}\right) d_{2}}}{i\left(k_{2 z}-k_{0} \cos \theta_{s}\right)} A_{2}^{\prime}\right.
\end{aligned}
$$

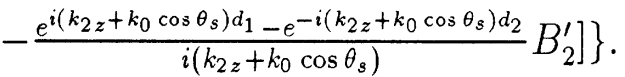

The SCPO approximation can also be obtained by noting that a magnetic current sheet of the form

$$
\mathbf{J}^{*}=-2 Z_{0} \cos \theta_{0} \Gamma^{\prime} e^{i k_{0} \sin \theta_{0} x} \delta(z) \hat{\mathbf{y}}
$$

generates a plane wave identical to the reflected wave. Using this as the equivalent surface current on the dielectric plate, the magnetic far field amplitude becomes

$$
\mathbf{S}_{H}^{S C P O}\left(\theta_{s}, \theta_{0}\right)=\hat{\mathbf{y}} \frac{-i k_{0}^{2}}{2 \pi} \cos \theta_{0} \Gamma^{\prime} a b \frac{\sin X}{X}
$$

As in the case of E polarization, the two approximations are identical in the specular direction, but (21) and (25) differ in all other directions, including backscattering $\left(\theta_{s}=\theta_{0}\right)$ unless $\theta_{0}=0$. 


\section{Numerical Results}

To illustrate the difference between the VIPO and SCPO approximations we consider a homogeneous (single layer) plate of thickness $d_{2}=\lambda_{0} / 4$ with $\epsilon_{2}=\epsilon_{1}=$ $3+i 0.1$. For an E-polarized plane wave incident at 30 degrees, the amplitude and phase of $\mathbf{S}^{V I P O} / \mathbf{S}^{S C P O}$ are given in Figs. 3 and 4 , and these show that the difference increases away from the specular and backscattering directions. At a fixed scattering angle, the difference increases with the electrical thickness of the plate up to the first resonance and then decreases. To test their accuracy the two approximations have been compared with the results of a moment method solution of the volume integral equation. The particular code used is a two-dimensional one which was extended to three dimensions by assuming that the induced currents are independent of the y coordinate. Since the dielectric constant of most vegetation materials is high, it is necessary to have the cell sizes very small, and one consequence of this is the need to compute the matrix elements extremely accurately, especially for $\mathrm{H}$ polarization. For a $2 \lambda_{0}$ square plate formed from the above-mentioned layer and illuminated by an E-polarized plane wave at normal incidence, the two approximations are compared with the moment method solution in Fig. 5, and the superiority of VIPO is clear.

In the case of a thin plate the two approximations are indistinguishable. This is illustrated in Fig. 6 showing the VIPO expression (14) and the moment method solution for a $2 \lambda_{0}$ square plate of thickness $d_{2}=\lambda_{0} / 50$ for $E$ polarization. The plate is a homogeneous one having $\epsilon=13+i 12$ corresponding to the average 
permittivity at $35 \mathrm{GHz}$ in Table 1 . The SCPO expression (18) yields the same results, as does a two-layer model having the permittivities listed in Table 1. The analogous data for $\mathrm{H}$ polarization are given in Fig. 7, and over a wide range of scattering angles, the approximate and moment method solutions are in excellent agreement.

As the frequency and, hence, the electrical thickness of the plate increase, the superiority of the VIPO approximation becomes apparent and, in addition, it becomes necessary to take the layering of the plate into account. In Figs. 8 and 9 the simulated frequency is $140 \mathrm{GHz}$, but to keep the moment method calculations tractable, the plate has been reduced in size to $1.4 \lambda_{0}$ by $2 \lambda_{0}$. The curves shown are for a two-layer plate having $d_{2}=2 d_{1}=0.5 \mathrm{~mm}$ with $\epsilon_{1}=5+i 4$ and $\epsilon_{2}=2+i 1$, and for a single layer having the average permittivity $\epsilon_{\text {avg }}=3.5+i 2.5$ (see Table 1). Since the accuracy of the physical optics approximation increases with the plate size, the agreement between the two-layer VIPO approximation and the moment method solution is remarkably good, and significantly better than if a single layer had been used.

\section{Conclusions}

A typical leaf has at least two dielectric layers whose cells have differing water content, and this produces a nonuniform dielectric profile which can now affect the scattering. At microwave frequencies where the leaf is no more than (about) $\lambda_{0} / 50$ in thickness, the nonuniformity is not important, and as shown by Senior et 
al [1987] the leaf can be modeled as a resistive sheet using an average value for the permittivity. If the physical optics approximation is then applied, the resulting scattering is attributed to a surface current, and this method is equivalent to the SCPO approximation. At higher frequencies, however, the thickness and structure of a leaf are more significant. At $100 \mathrm{GHz}$ and above a leaf is a considerable fraction of a wavelength in thickness, and in spite of the reduced sensitivity to water content, the nonuniformity affects the scattering.

For a two-layer model of a leaf, the SCPO approximation has been compared with the volume integral (VIPO) approximation. When the leaf is thin the two approximations are identical and in good agreement with data obtained from a moment method solution of the integral equation, but as the electrical thickness increases, the two approximations diverge in all directions except the specular and (for E polarization) backscattering ones. Although the VIPO approximation is more complicated, its accuracy is greater, and the agreement with the moment method data is better using a two-layer model than when a single layer of average permittivity is employed.

For most practical purposes it would appear that VIPO in conjunction with an accurate dielectric profile of a leaf provides an adequate approximation to the scattering at millimeter wavelengths. As our knowledge of the profile increases, it may be desirable to use a multi-layer model which could even simulate a continuous, nonuniform profile, and a convenient way of doing this is described in the Appendix. We also note that at frequencies for which the leaf thickness is comparable to $\lambda_{m} / 2$ 
where $\lambda_{m}$ is the (average) wavelength in the leaf, the scattering is greatly reduced at some angle of incidence, and because the permittivity is complex, there is actually a range of angles for which this is true. Since the reduction is accompanied by an increase in the field transmitted through the leaf, this could provide a means for penetration through a vegetation canopy.

\section{Acknowledgement}

This work was supported by the U.S. Army Research Office under contract DAAG $29-85-\mathrm{k}-0220$. 


\section{References}

[1 ] Le Vine, D.M., A. Snyder, R.H. Lang, and H.G. Garter, Scattering from thin dielectric disks, IEEE Trans. Antennas Propag., 33, 1410-1413, 1985.

[2 ] Sarabandi, K., T.B.A. Senior, and F.T. Ulaby, Effect of curvature on the backscattering from a leaf, J. Electromag. Waves and Applics., 2, 653-670, 1988.

[3 ] Senior, T.B.A., K. Sarabandi, and F.T. Ulaby, Measuring and modeling the backscattering cross section of a leaf, Radio Sci., 22, 1109-1116, 1987.

[4 ] Senior, T.B.A., and J.L. Volakis, Sheet simulation of a thin dielectric layer, Radio Sci., 22, 1261-1272, 1987.

[5 ] Willis, T.M., H. Weil, and D.M. Le Vine, Applicability of physical optics thin plate scattering formulas for remote sensing, IEEE Trans. Geosci. Remote Sensing, 26, 153-160, 1988. 


\section{Appendix A}

\section{A1 Combined Sheets Model}

When using the VIPO approximation, an efficient way to take into account the effect of any non-uniformity in the dielectric profile is to model the leaf as a stack of $N$ combined current sheets. Each sheet simulates a very thin dielectric layer whose thickness is less than $\lambda / 15$ where $\lambda$ is the wavelength in the material. A combined sheet consists of coincident resistive and modified conductive sheets that support electric and magnetic currents respectively, with the conductive sheet accounting for the electric currents flowing perpendicular to the dielectric layer. The $m^{\text {th }}$ layer sheets are characterized by a complex resistivity and conductivity $R_{m}$ and $R_{m}^{*}$, respectively, where

$$
\begin{aligned}
R_{m} & =\frac{i Z_{0}}{k_{0} \Delta_{m}\left(\epsilon_{m}-1\right)} \\
R_{m}^{*} & =\frac{i Y_{0} \epsilon_{m}}{k_{0} \Delta_{m}\left(\epsilon_{m}-1\right)} .
\end{aligned}
$$

Here $\epsilon_{m}$ and $\Delta_{m}$ are the relative dielectric constant and thickness of the $m^{\text {th }}$ layer, and $\tau=\sum_{m=1}^{N} \Delta_{m}$ is the total thickness of the dielectric slab.

The boundary conditions at the $m^{\text {th }}$ combined sheet are as follows [Senior and Volakis; 1987]:

$$
\begin{array}{r}
\hat{\mathbf{n}} \times\left\{\hat{\mathbf{n}} \times\left[\mathbf{E}^{+}+\mathbf{E}^{-}\right]\right\}=-2 R_{m} \mathbf{J}_{m} \\
\mathbf{J}_{m}=\hat{\mathbf{n}} \times\left[\mathbf{H}^{+}-\mathbf{H}^{-}\right]
\end{array}
$$


where $\mathbf{J}_{m}$ is the total electric current supported by the resistive sheet, and

$$
\begin{gathered}
\hat{\mathbf{n}} \times\left\{\hat{\mathbf{n}} \times\left[\mathbf{H}^{+}+\mathbf{H}^{-}\right]\right\}-\frac{i Y_{0}}{k_{0}} \hat{\mathbf{n}} \times \frac{\partial}{\partial n}\left[\mathbf{E}^{+}+\mathbf{E}^{-}\right]=-2 R_{m}^{*} \mathbf{J}_{m}^{*} \\
\mathbf{J}_{m}^{*}=-\hat{\mathbf{n}} \times\left[\mathbf{E}^{+}-\mathbf{E}^{-}\right]
\end{gathered}
$$

where $\mathbf{J}_{m}^{*}$ is the total magnetic current supported by the conductive sheet. The superscripts ${ }^{+,-}$refer to the upper $(+)$and lower $(-)$sides of the sheet, and $\hat{\mathrm{n}}$ is the unit vector outward normal to the upper side.

\section{A2 Scattering by a Stack of N Planar Sheets}

Consider a stack of $N$ infinite planar combined sheets all parallel to the $x y$ plane of a Cartesian coordinate system $(x, y, z)$ as depicted in Fig. A1. The top sheet is in the $z=0$ plane and the $m^{\text {th }}$ sheet is located at $z=-d_{m}$,

where $d_{1}=0$. The space between the $m^{\text {th }}$ and $(m+1)^{t h}$ sheets is referred to as region $m$, and we note that region $0(z>0)$ and region $N\left(z<-d_{n}\right)$ are semi-infinite free space. A plane wave whose plane of incidence is parallel to the $x z$ plane impinges on the stack of sheets from above. From the symmetry of the problem, all the field vectors are independent of $y$ (i.e., $\frac{\partial}{\partial y}=0$ ), as a result of which the field components in each region can be separated into $E-$ and $H$-polarized waves which are the dual of each other.

In the case of $\mathrm{E}$ polarization the incident field is given by (1) and the field 
components in region $m$ can be expressed as

$$
\begin{aligned}
& E_{m y}=\left[C_{m}^{i} e^{-i k_{0} \cos \theta_{0} z}+C_{m}^{r} e^{i k_{0} \cos \theta_{0} z}\right] e^{i k \sin \theta_{0} x} \\
& H_{m x}=Y_{0} \cos \theta_{0}\left[C_{m}^{i} e^{-i k_{0} \cos \theta_{0} z}-C_{m}^{r} e^{i k_{0} \cos \theta_{0} z}\right] e^{i k_{0} \sin \theta_{0} x} \\
& H_{m z}=Y_{0} \sin \theta_{0}\left[C_{m}^{i} e^{-i k_{0} \cos \theta_{0} z}+C_{m}^{r} e^{i k_{0} \cos \theta_{0} z}\right] e^{i k_{0} \sin \theta_{0} x}
\end{aligned}
$$

The coefficients $C_{m}^{i}$ and $C_{m}^{r}$ are the amplitudes of the waves travelling in the $-z$ and $+z$ directions, respectively, in region $m$. In region $0, C_{0}^{i}=1$ and $C_{0}^{r}=\Gamma_{E}$ (the total reflection coefficient) and in region $N, C_{N}^{r}=0$, $C_{N}^{i}=T_{E}$ (the total transmission coefficient). Hence, using the boundary conditions (A2)-(A5), there are $2 N$ unknowns and $2 N$ equations that can be solved simultaneously. On substitution of (A6) into (A4) the left hand side vanishes showing $\mathbf{J}_{m}^{*}=0$. As expected, the conductive sheet is not excited with this polarization since there is no current in the $z$ direction in the dielectric slab, and in the absence of a magnetic source, the tangential component of the electric field must be continuous as given by (A5). On inserting the expressions (A6) into (A5) and (A2) and defining the reflection coefficient in region $m$ as

$$
\Gamma_{m}^{E}=\frac{C_{m}^{r}}{C_{m}^{i}} e^{-2 i k_{0} \cos \theta_{0} d_{m+1}}
$$

the following relations are obtained:

$$
\begin{aligned}
& \Gamma_{m-1}^{E}=\frac{-1+\left(2 Y_{0} \cos \theta_{0} R_{m-1}\right) e^{2 i k_{0} \cos \theta_{0}\left(d_{m+1}-d_{m}\right)} \Gamma_{m}^{E}}{1+2 Y_{0} \cos \theta_{0} R_{m}+e^{2 i k_{0} \cos \theta_{0}\left(d_{m+1}-d_{m}\right)} \Gamma_{m}^{E}} \\
& C_{m}^{i}=\frac{1+\Gamma_{m-1}^{E}}{1+e^{2 i k_{0} \cos \theta_{0}\left(d_{m+1}-d_{m}\right)} \Gamma_{m}^{E}} C_{m-1}^{i}
\end{aligned}
$$

The induced electric current in the $m^{\text {th }}$ sheet can be found from (A3) and 
expressed as (excluding the phase factor $e^{i k_{0} \sin \theta_{0} x}$ )

$$
\begin{aligned}
\mathbf{J}_{m}^{E}= & \hat{\mathbf{y}} 2 Y_{0} \cos \theta_{0} e^{i k_{0} \cos \theta_{0} d_{m}}\left[1-\frac{1+\Gamma_{m}^{E}}{1+e^{2 i k_{0} \cos \theta_{0}\left(d_{m}+d_{m}\right)} \Gamma_{m}^{E}}\right] \\
& \cdot \prod_{\ell=1}^{m-1}\left(\frac{1+\Gamma_{\ell-1}^{E}}{1+e^{2 i k_{0} \cos \theta_{0}\left(d_{\ell+1}-d_{\ell}\right)} \Gamma_{\ell}^{E}}\right) .
\end{aligned}
$$

The total reflection coefficient in region $0\left(\Gamma_{E}(\theta)=\Gamma_{0}^{E}\right)$ can be evaluated from the recursive relation (A7) by noting that $\Gamma_{N}^{E}=0$ (the region $N$ is semi-infinite). The total transmission coefficient can also be obtained from (A7) as follows:

$$
T_{E}(\theta)=\frac{C_{N}^{i}}{C_{0}^{i}}=\prod_{m=1}^{N}\left[\frac{1+\Gamma_{m-1}^{E}}{1+e^{2 i k_{0} \cos \theta_{0}\left(d_{m+1}-d_{m}\right)} \Gamma_{m}^{E}}\right]
$$

Unlike the E-polarized case where the magnetic current is zero, an $\mathrm{H}$-polarized wave excites a magnetic current in the $y$ direction and the tangential electric and magnetic fields are both discontinuous across the combined sheets. For $\mathrm{H}$ polarization the tangential field vectors in region $m$ can be obtained by applying the duality relationships to (A6). In this case the amplitudes of the travelling in $-z$ and $+z$ directions are denoted by $B_{m}^{i}$ and $B_{m}^{r}$ respectively. By applying the boundary conditions (A2)-(A5) at the $m^{\text {th }}$ sheet and denoting the reflection coefficient in region $m$ by

$$
\Gamma_{m}^{H}=\frac{B_{m}^{r}}{B_{m}^{i}} e^{-2 i k_{0} \cos \theta_{0} d_{m+1}}
$$

after some algebraic manipulation we obtain

$$
\begin{aligned}
\Gamma_{m-1}^{H} & =\frac{\left(Q_{m} P_{m}-1\right)-\left(1-P_{m}\right)\left(Q_{m}-1\right) \Gamma_{m}^{H} e^{2 i k_{0} \cos \theta_{0}\left(d_{m+1}-d_{m}\right)}}{-\left(1+P_{m}\right)\left(1+Q_{m}\right)+\left(1-Q_{m} P_{m}\right) \Gamma_{m}^{H} e^{2 i k_{0} \cos \theta_{0}\left(d_{m+1}-d_{m}\right)}} \\
B_{m}^{i} & =\frac{\left(Q_{m}-1\right)+\left(1+Q_{m}\right) \Gamma_{m-1}^{H}}{-\left(1+Q_{m}\right)+\left(1-Q_{m}\right) \Gamma_{m}^{H} e^{2 i k_{0} \cos \theta_{0}\left(d_{m+1}-d_{m}\right)}} B_{m-1}^{i}
\end{aligned}
$$


where the parameters $Q_{m}$ and $P_{m}$ are

$$
Q_{m}=\frac{\sin ^{2} \theta_{0}}{2 R_{m}^{*} Z_{0} \cos \theta_{0}} \quad P_{m}=\frac{2 R_{m} \sec \theta_{0}}{Z_{0}}
$$

Since $\Gamma_{N}^{H}=0$ the recursive relation (A11) gives the total reflection coefficient in region 0 and using (A12) the total transmission coefficient is given by

$$
T_{H}(\theta)=\prod_{m=1}^{N}\left[\frac{\left(Q_{m}-1\right)+\left(1+Q_{m}\right) \Gamma_{m-1}^{H}}{-\left(1+Q_{m}\right)+\left(1-Q_{m}\right) \Gamma_{m}^{H} e^{2 i k_{0} \cos \theta_{0}\left(d_{m+1}-d_{m}\right)}}\right]
$$

The induced electric and magnetic currents can be expressed in terms of the reflection coefficients as

$$
\begin{aligned}
\mathbf{J}_{m}^{H}= & -\hat{\mathbf{x}} e^{i k_{0} \cos \theta_{0} d_{m-1}}\left[\left(1+\Gamma_{m-1}^{H}\right)+\left(1+\Gamma_{m}^{H} e^{2 i k_{0} \cos \theta_{0}\left(d_{m+1}-d_{m}\right)}\right)\right. \\
& \left.\cdot \frac{\left(Q_{m}-1\right)+\left(1+Q_{m}\right) \Gamma_{m-1}^{H}}{\left(1+Q_{m}\right)+\left(1-Q_{m}\right) \Gamma_{m}^{H} e^{i k \cos \theta_{0}\left(d_{m+1}-d_{m}\right)}}\right] \cdot \prod_{\ell=1}^{m-1}\left[\frac{\left(Q_{\ell}-1\right)+\left(1+Q_{\ell}\right) \Gamma_{\ell-1}^{H}}{\left(1+Q_{\ell}\right)+\left(1-Q_{\ell}\right) \Gamma_{\ell}^{H} e^{i k_{0} \cos \theta_{0}\left(d_{\ell+1}-d_{\ell}\right)}}\right] \\
\mathbf{J}_{m}^{H *}= & \hat{\mathrm{y}} Z_{0} \cos \theta_{0} \cdot e^{i k_{0} \cos \theta_{0} d_{m-1}}\left[\left(1-\Gamma_{m-1}^{H}\right)+\left(1-\Gamma_{m}^{H} e^{2 i k_{0} \cos \theta_{0}\left(d_{m+1}-d_{m}\right)}\right)\right. \\
& \left.\cdot \frac{\left(Q_{m}-1\right)+\left(1+Q_{m}\right) \Gamma_{m-1}^{H}}{\left(1+Q_{m}\right)+\left(1-Q_{m}\right) \Gamma_{m}^{H} e^{i k \cos \theta_{0}\left(d_{m+1}-d_{m}\right)}}\right] \cdot \prod_{\ell=1}^{m-1}\left[\frac{\left(Q_{\ell}-1\right)+\left(1+Q_{\ell}\right) \Gamma_{\ell-1}^{H}}{\left(1+Q_{\ell}\right)+\left(1-Q_{\ell}\right) \Gamma_{\ell}^{H} e^{i k_{0} \cos \theta_{0}\left(d_{\ell+1}-d_{\ell}\right)}}\right]
\end{aligned}
$$

where $\mathbf{J}_{m}^{H}(x)=\mathbf{J}_{m}^{H} e^{i k_{0} \sin \theta_{0} x}$ and $\mathbf{J}_{m}^{H *}(x)=\mathbf{J}_{m}^{H *} e^{i k_{0} \sin \theta_{0} x}$

\section{A3 Scattering by a Rectangular Stack}

Consider a portion of the N-layered stack of combined sheets in the form of

a rectangle occupying the region $-\frac{a}{2} \leq x \leq \frac{a}{2},-\frac{b}{2} \leq y \leq \frac{b}{2}$ as depicted in

Fig. A2. In the far zone the approximation

$$
\left|\bar{r}-\bar{r}_{m}^{\prime}\right| \approx r+\sin \theta_{s} x^{\prime}+\cos \theta_{s} d_{m}
$$


leads to

$$
\begin{aligned}
& \Pi(\bar{r}) \approx \frac{e^{i k_{0} r}}{k_{0} r} \frac{i Z_{0}}{4 \pi} \int_{-a / 2}^{a / 2} \int_{-b / 2}^{b / 2}\left(\sum_{m=1}^{N} \mathbf{J}_{m}\left(\bar{r}^{\prime}\right) e^{i k_{0} \cos \theta_{s} d_{m}}\right) e^{i k_{0} \sin \theta_{s} x^{\prime}} \mathrm{d} x^{\prime} \mathrm{d} y^{\prime}, \\
& \Pi^{*}(\bar{r}) \approx \frac{e^{i k_{0} r}}{k_{0} r} \frac{i Y_{0}}{4 \pi} \int_{-a / 2}^{a / 2} \int_{-b / 2}^{b / 2}\left(\sum_{m=1}^{N} \mathbf{J}_{m}^{*}\left(\bar{r}^{\prime}\right) e^{i k_{0} \cos \theta_{s} d_{m}}\right) e^{i k_{0} \sin \theta_{s} x^{\prime}} \mathrm{d} x^{\prime} \mathrm{d} y^{\prime} .
\end{aligned}
$$

Using the physical optics approximation, the currents obtained for the infinite sheets are substituted into (A16) to find the scattered fields. For E and H polarizations the far field amplitudes are

$$
\begin{gathered}
\mathbf{S}_{E}\left(\theta_{s}, \theta_{0}\right)=\hat{\mathbf{y}} \frac{i}{4 \pi} k_{0}^{2} a b Z_{0}\left(\sum_{m=1}^{N} \mathbf{J}_{m}^{E} e^{i k_{0} \cos \theta_{s} d_{m}}\right) \frac{\sin X}{X} \\
\mathbf{S}_{H}\left(\theta, \theta_{0}\right)=\hat{\mathbf{y}} \frac{i}{4 \pi} k_{0}^{2} a b\left[\sum_{m=1}^{N}\left(\cos \theta_{s} \mathbf{J}_{m}^{H}+Y_{0} \mathbf{J}_{m}^{H *}\right) e^{i k_{0} \cos \theta_{s} d_{m}}\right] \frac{\sin X}{X} .
\end{gathered}
$$

where, as before, $X=\frac{k a}{2}\left(\sin \theta_{s}+\sin \theta_{0}\right)$. In the backscattering $\left(\theta_{s}=\theta_{0}\right)$ and specular $\left(\theta_{s}=-\theta_{0}\right)$ directions the summation term in (A17) reduces to a telescopic series resulting in

$$
\sum_{m=1}^{N} \mathbf{J}_{m}^{E} e^{i k \cos \theta_{0} d_{m}}=2 Y_{0} \cos \theta_{0} C_{0}^{r}=2 Y_{0} \cos \theta_{0} \Gamma_{E}\left(\theta_{0}\right),
$$

and backscattering cross section is then

$$
\sigma_{E}\left(\theta_{0}, \theta_{0}\right)=4 \pi \frac{(a b)^{2}}{\lambda^{2}} \cos ^{2} \theta_{0}\left|\Gamma_{E}\left(\theta_{0}\right)\right|^{2} \frac{\sin ^{2}\left(k a \sin \theta_{0}\right)}{\left(k a \sin \theta_{0}\right)^{2}}
$$

Also, for $\mathrm{H}$ polarization

$$
\begin{aligned}
\sum_{m=1}^{N}\left(\cos \theta_{0} J_{m}^{H}+Y_{0} J_{m}^{H *}\right) e^{i k_{0} \cos \theta_{0} d_{m}}= & -2 \cos \theta_{0} \sum_{m=1}^{N}\left(B_{m-1}^{r}-B_{m}^{r}\right) \\
& =-2 \cos \theta_{0} B_{0}^{r}=-2 \cos _{\theta_{0}} \Gamma_{H}\left(\theta_{0}\right),
\end{aligned}
$$


which leads to

$$
\sigma_{H}\left(\theta_{0}, \theta_{0}\right)=4 \pi \frac{(a b)^{2}}{\lambda^{2}} \cos ^{2} \theta_{0}\left|\Gamma_{H}\left(\theta_{0}\right)\right|^{2} \frac{\sin ^{2}\left(k a \sin \theta_{0}\right)}{\left(k a \sin \theta_{0}\right)^{2}}
$$

These results are identical with the ones obtained by the SCPO technique. The extinction cross section can also be obtained from the far field amplitude using

$$
\sigma^{e x t}=\frac{\lambda^{2}}{\pi} \operatorname{Im}\left[\mathbf{S}\left(\theta_{0}, \theta_{0}+\pi\right)\right]
$$

from which we obtain

$$
\begin{aligned}
\sigma_{E}^{e x t} & =2 a b \cos \theta_{0} \operatorname{Re}\left[1-T_{E}(\theta)\right] \\
\sigma_{H}^{e x t} & =2 a b \cos \theta_{0} \operatorname{Re}\left[1-T_{H}\left(\theta_{0}\right)\right] .
\end{aligned}
$$




\section{List of Figures}

1 The structure of a typical vegetation leaf. . . . . . . . . . . 22

2 The geometry of the scattering of a plane wave from a two-layer dielectric slab. . . . . . . . . . . . . . . 22

3 Amplitude of the ratio of the bistatic far field amplitude of VIPO to SCPO for E polarization of a dielectric plate with $d_{2}=\lambda_{0} / 4$ and $\epsilon_{1}=\epsilon_{2}=3+i 0.1$ at $\theta_{0}=30$ degrees. . . . . . . . . .

4 Phase of the ratio of the bistatic far field amplitude of VIPO to SCPO for E polarization of a dielectric plate with $d_{2}=\lambda_{0} / 4$ and $\epsilon_{1}=\epsilon_{2}=3+i 0.1$ at $\theta_{0}=30$ degrees. . . . . . . . . . 24

5 The bistatic cross section of a $2 \lambda_{0} \times 2 \lambda_{0}$ plate for E polarization with $d_{2}=\lambda_{0} / 4$ and $\epsilon_{1}=\epsilon_{2}=3+i 0.1$ at normal incidence: $(-)$ moment method solution, (- - ) VIPO, (- -) SCPO. . . . . . . . 25

6 The bistatic cross section area of a $2 \lambda_{0} \times 2 \lambda_{0}$ plate for $E$ polarization with $d_{2}=\lambda_{0} / 50$ and $\epsilon_{a v g}=13+i 12$ at normal incidence: $(-)$ moment method solution, (- - ) VIPO or SCPO. . . . . . . . . 26

7 The bistatic cross section of a $2 \lambda_{0} \times 2 \lambda_{0}$ plate for $H$ polarization with $d_{2}=\lambda_{0} / 50$ and $\epsilon_{a v g}=13+i 12$ at normal incidence: $(-)$ moment method solution, (- -) VIPO or SCPO. 
8 The bistatic cross section of a $1.4 \lambda_{0} \times 2 \lambda_{0}$ plate for $E$ polarization with $d_{2}=2 d_{1}=0.5 \mathrm{~mm}$ and $f=140 \mathrm{GHz}$ at normal incidence: $(-$ ) moment method solution with $\epsilon_{1}=5+i 4, \epsilon_{2}=2+i 1,(--)$ VIPO with $\epsilon_{1}=5+i 4, \epsilon_{2}=2+i 1,(--)$ VIPO with $\epsilon_{2}=\epsilon_{1}=3.5+i 2.5 . \quad 28$

9 The bistatic cross section area of a $1.4 \lambda_{0} \times 2 \lambda_{0}$ plate for $\mathrm{H}$ polarization with $d_{2}=2 d_{1}=0.5 \mathrm{~mm}$ and $f=140 \mathrm{GHz}$ at normal incidence: (-) moment method solution with $\epsilon_{1}=5+i 4, \epsilon_{2}=2+i 1,(--)$ VIPO with $\epsilon_{1}=5+i 4 \epsilon_{2}=2+i 1,(--)$ VIPO with $\epsilon_{2}=\epsilon_{1}=3.5+i 2.5 .29$

A-1 Layer of $\mathrm{N}$ combined sheets simulating infinite dielectric slab. . . . 30

A-2 The geometry of the scattering of a plane wave from a finite N-layer combined-sheet. . . . . . . . . . . . . . . 30 


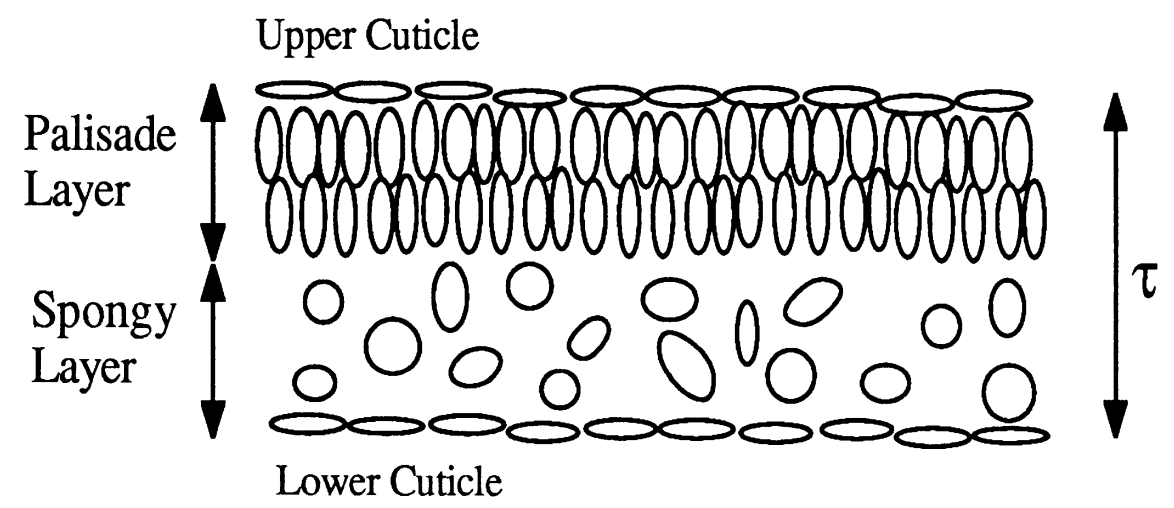

Figure 1: The structure of a typical vegetation leaf.

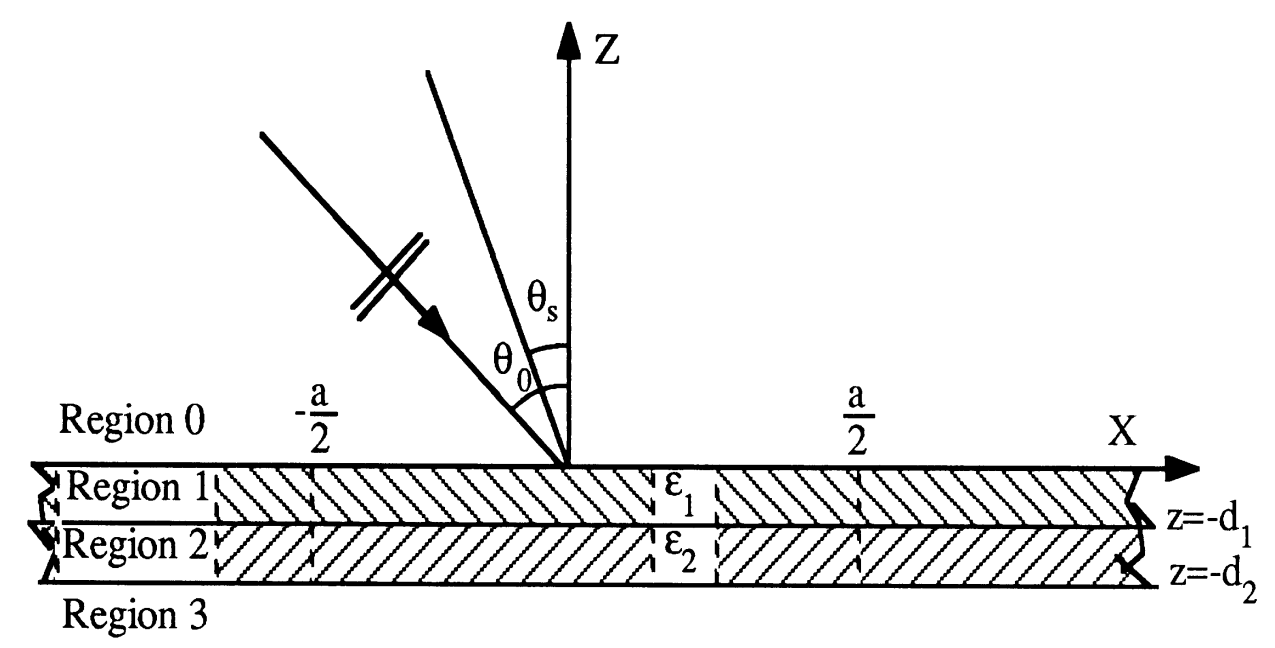

Figure 2: The geometry of the scattering of a plane wave from a two-layer dielectric slab. 


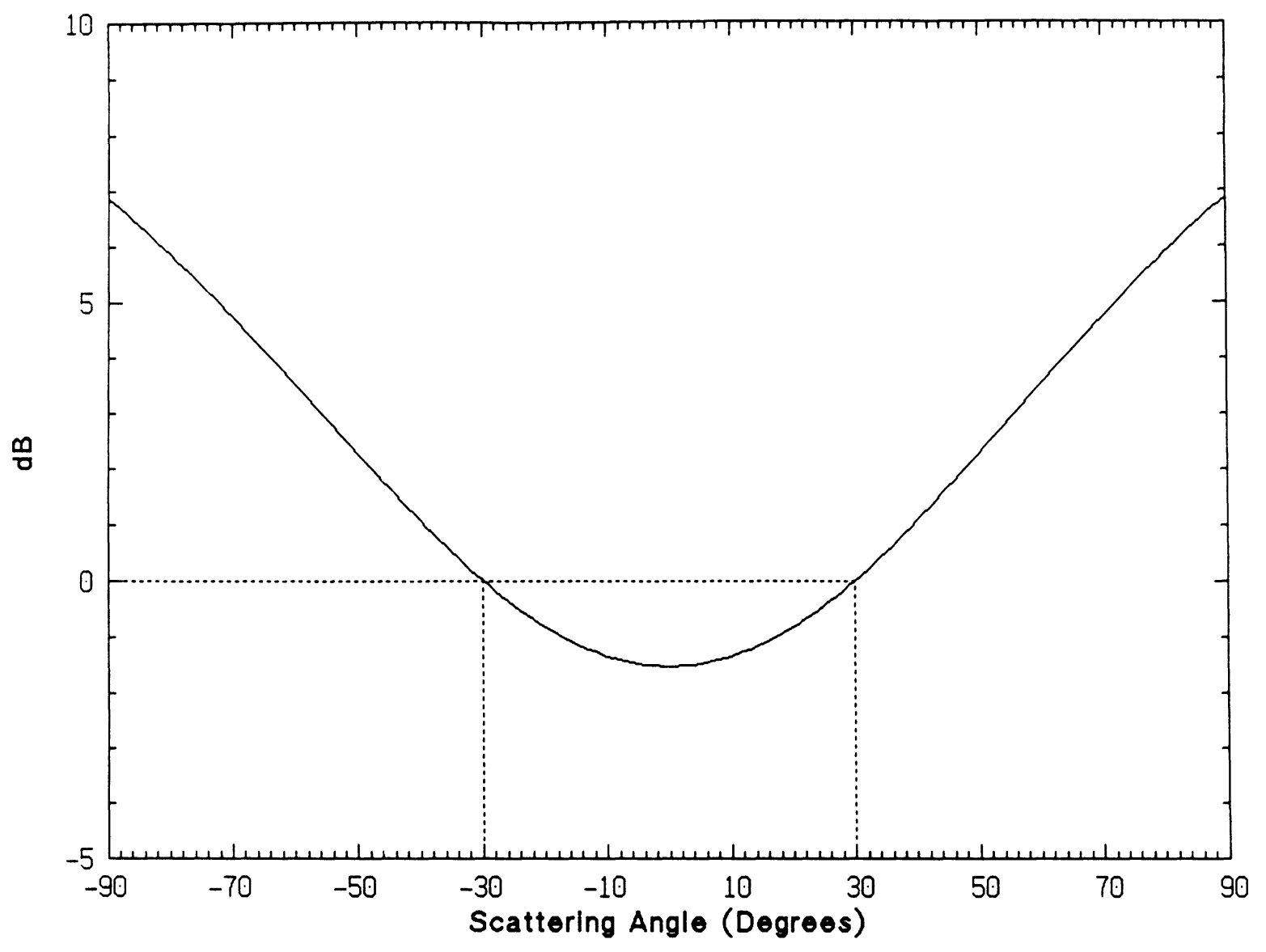

Figure 3: Amplitude of the ratio of the bistatic far field amplitude of VIPO to SCPO for E polarization of a dielectric plate with $d_{2}=\lambda_{0} / 4$ and $\epsilon_{1}=\epsilon_{2}=3+i 0.1$ at $\theta_{0}=30$ degrees. 


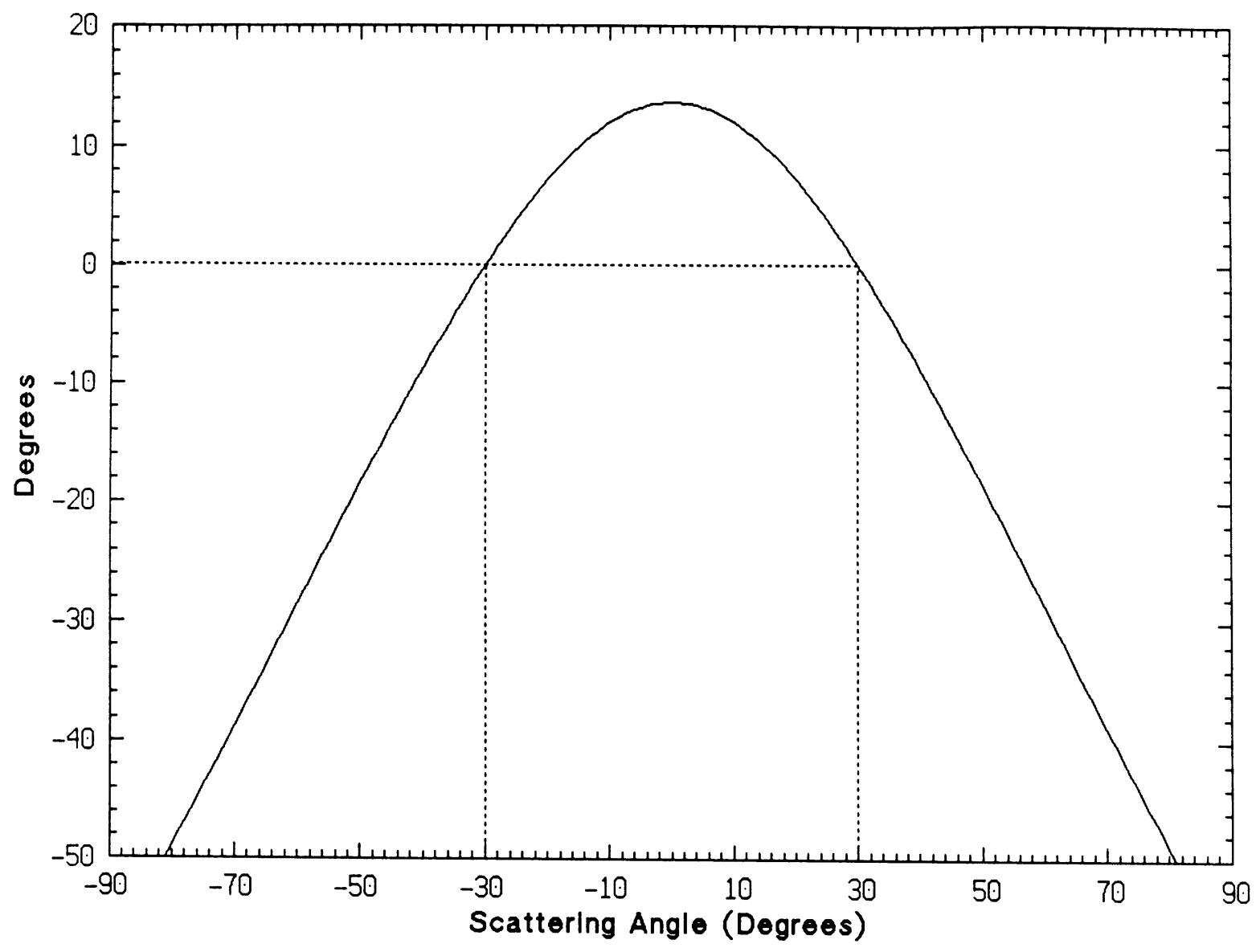

Figure 4: Phase of the ratio of the bistatic far field amplitude of VIPO to SCPO for $\mathrm{E}$ polarization of a dielectric plate with $d_{2}=\lambda_{0} / 4$ and $\epsilon_{1}=\epsilon_{2}=3+i 0.1$ at $\theta_{0}=30$ degrees. 


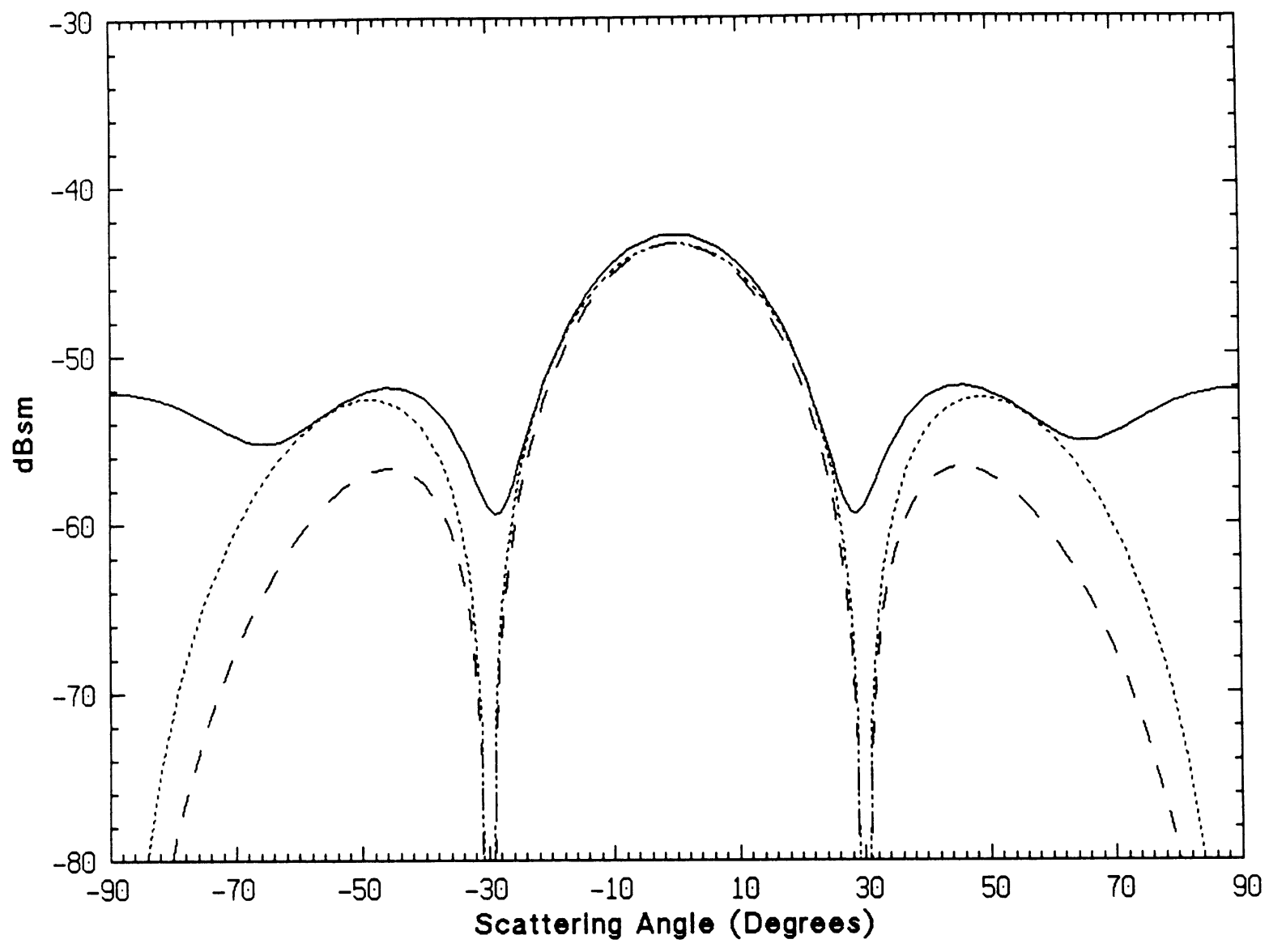

Figure 5: The bistatic cross section of a $2 \lambda_{0} \times 2 \lambda_{0}$ plate for E polarization with $d_{2}=\lambda_{0} / 4$ and $\epsilon_{1}=\epsilon_{2}=3+i 0.1$ at normal incidence: $(-)$ moment method solution, (- - ) VIPO, (- -) SCPO. 


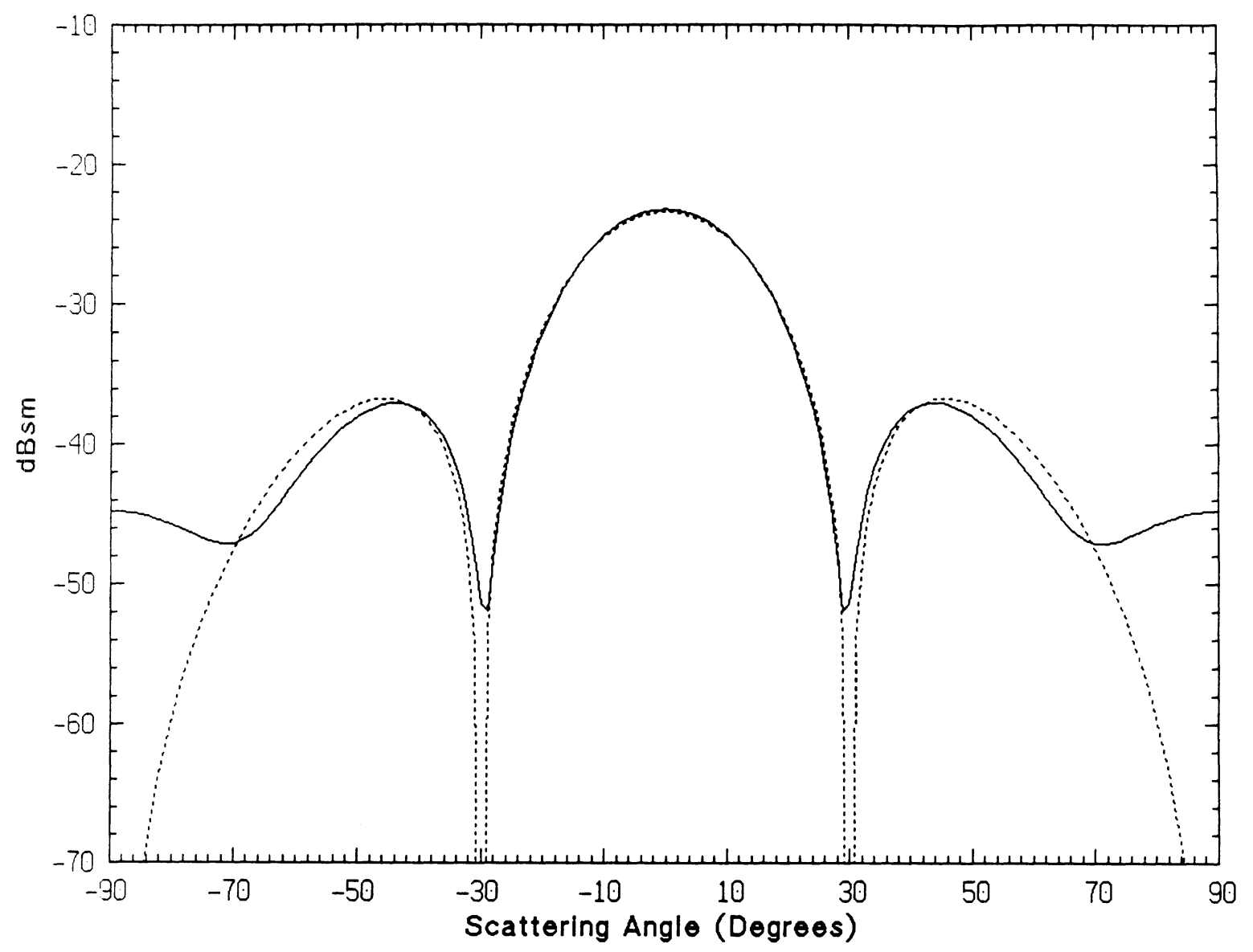

Figure 6: The bistatic cross section area of a $2 \lambda_{0} \times 2 \lambda_{0}$ plate for E polarization with $d_{2}=\lambda_{0} / 50$ and $\epsilon_{a v g}=13+i 12$ at normal incidence: $(-)$ moment method solution, (- -) VIPO or SCPO. 


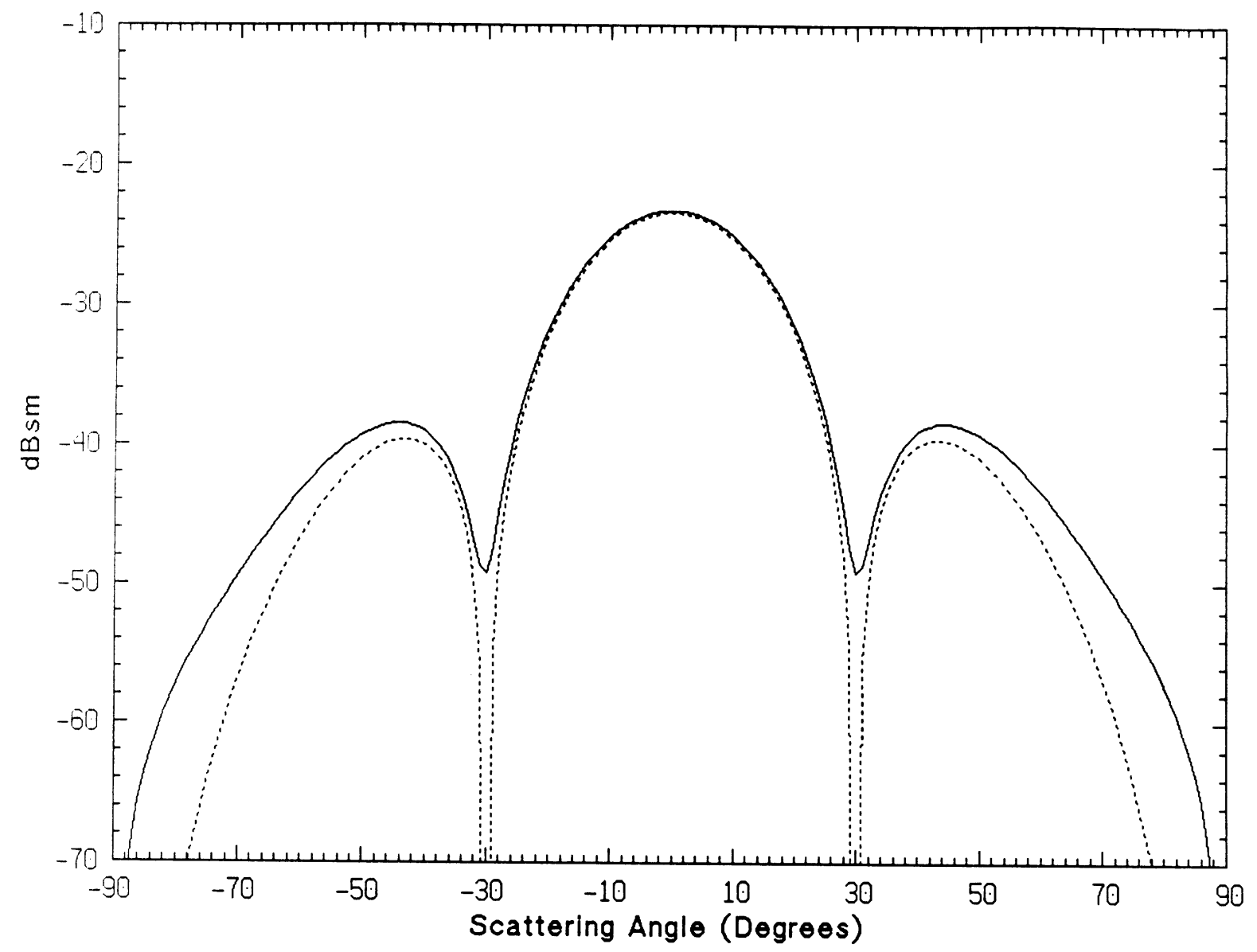

Figure 7: The bistatic cross section of a $2 \lambda_{0} \times 2 \lambda_{0}$ plate for $\mathrm{H}$ polarization with $d_{2}=\lambda_{0} / 50$ and $\epsilon_{a v g}=13+i 12$ at normal incidence: $(-)$ moment method solution, (- - ) VIPO or SCPO. 


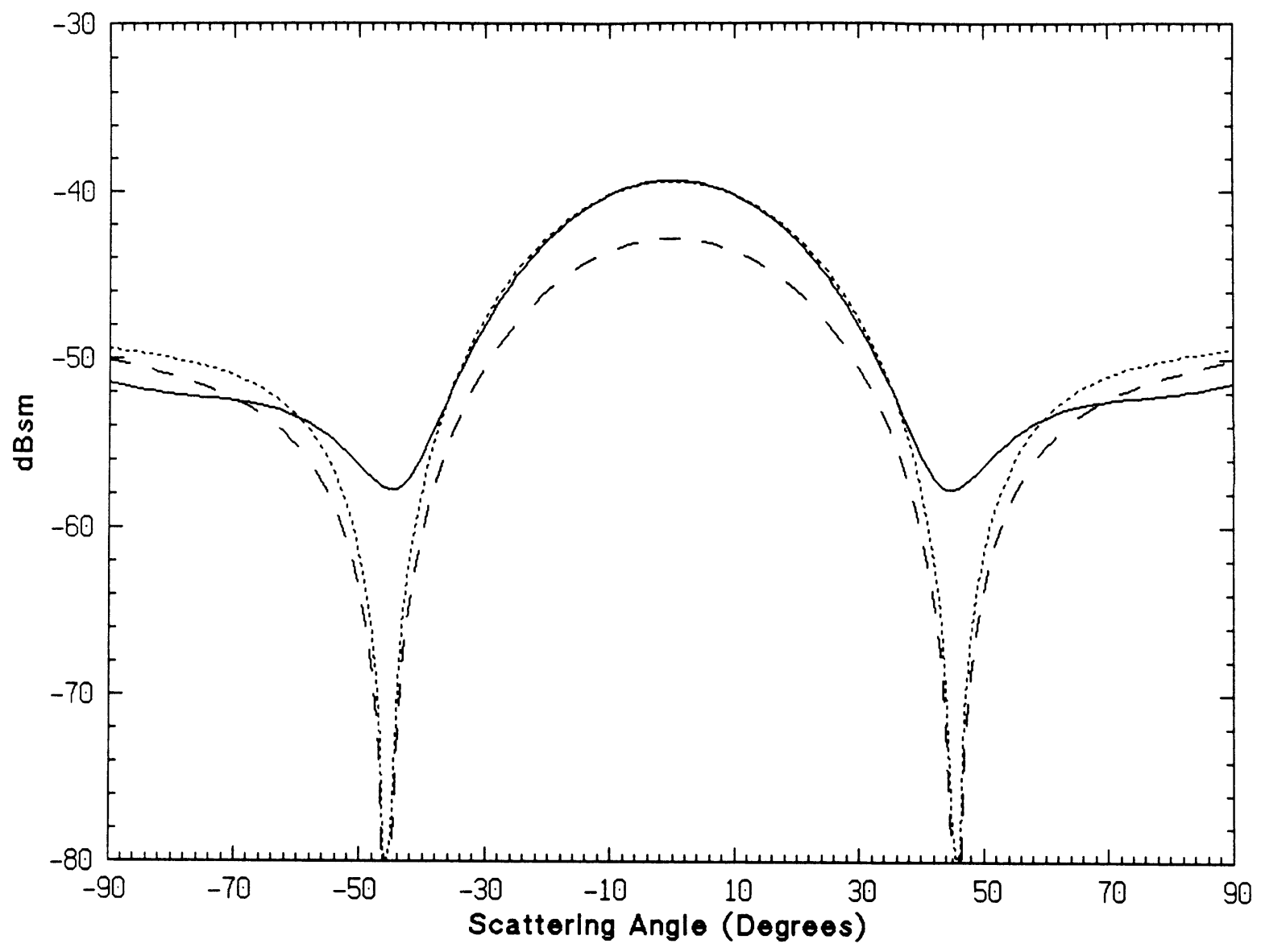

Figure 8: The bistatic cross section of a $1.4 \lambda_{0} \times 2 \lambda_{0}$ plate for $\mathrm{E}$ polarization with $d_{2}=2 d_{1}=0.5 \mathrm{~mm}$ and $f=140 \mathrm{GHz}$ at normal incidence: $(-)$ moment method solution with $\epsilon_{1}=5+i 4, \epsilon_{2}=2+i 1,(--)$ VIPO with $\epsilon_{1}=5+i 4, \epsilon_{2}=2+i 1$, $(--)$ VIPO with $\epsilon_{2}=\epsilon_{1}=3.5+i 2.5$. 


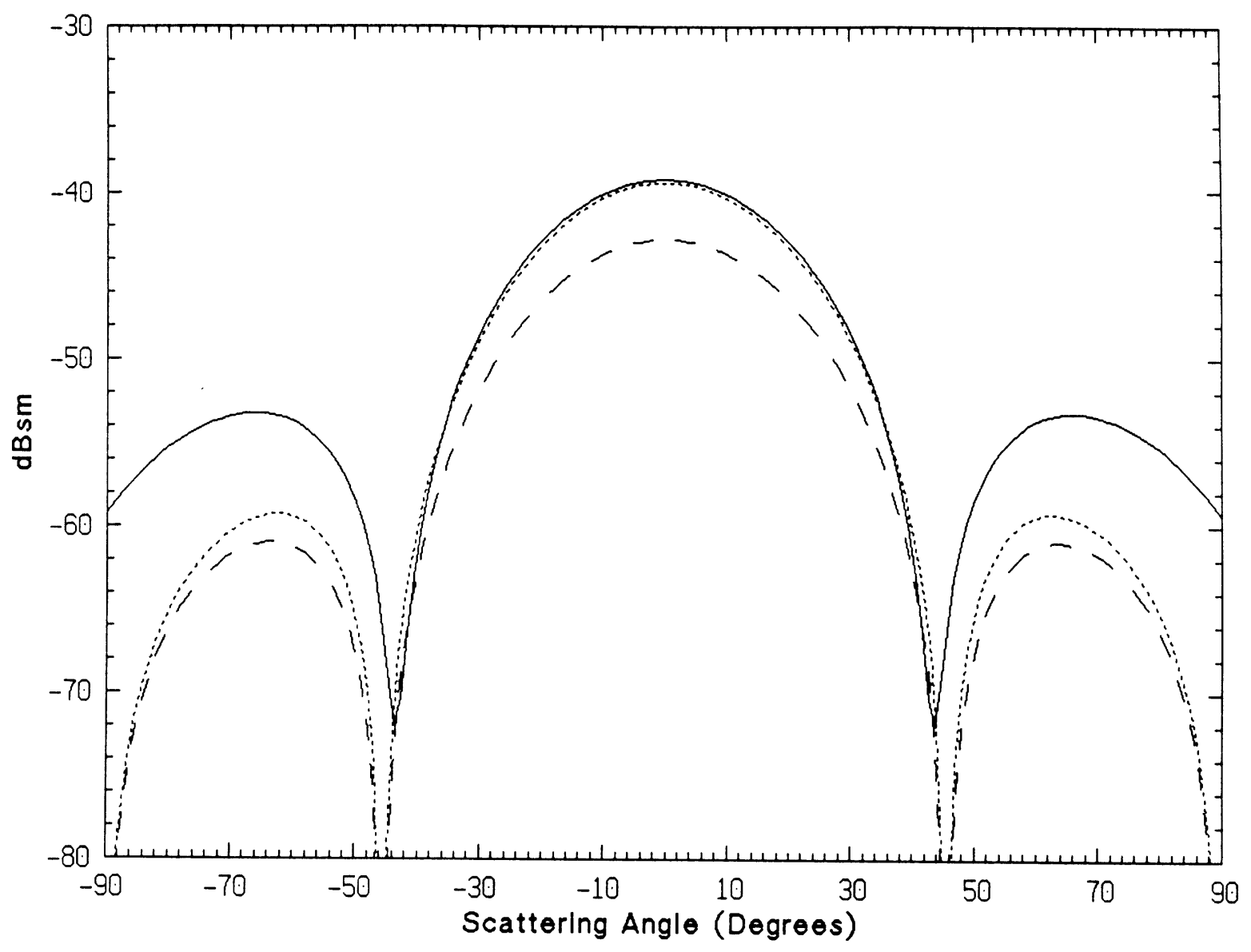

Figure 9: The bistatic cross section area of a $1.4 \lambda_{0} \times 2 \lambda_{0}$ plate for $\mathrm{H}$ polarization with $d_{2}=2 d_{1}=0.5 \mathrm{~mm}$ and $f=140 \mathrm{GHz}$ at normal incidence: (-) moment method solution with $\epsilon_{1}=5+i 4, \epsilon_{2}=2+i 1,\left(-\right.$ - ) VIPO with $\epsilon_{1}=5+i 4$ $\epsilon_{2}=2+i 1,(--)$ VIPO with $\epsilon_{2}=\epsilon_{1}=3.5+i 2.5$. 


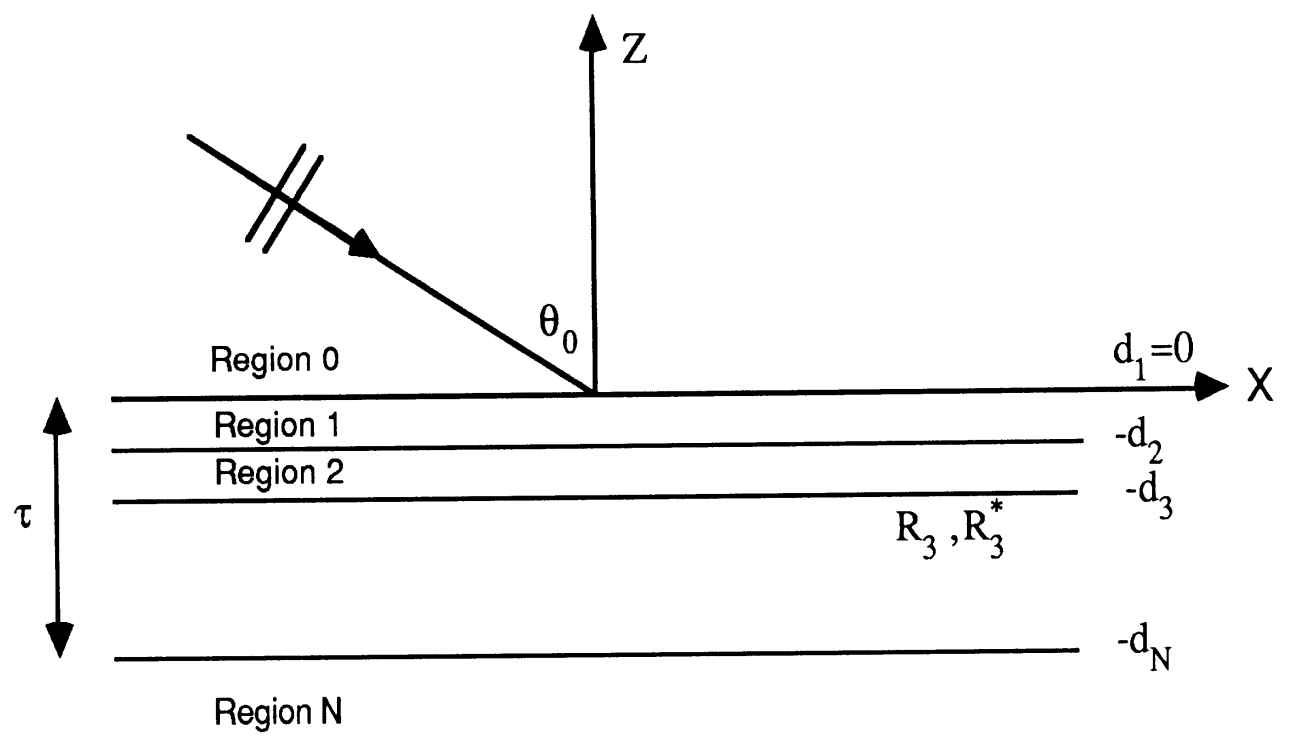

Figure A-1: Layer of $\mathrm{N}$ combined sheets simulating infinite dielectric slab.

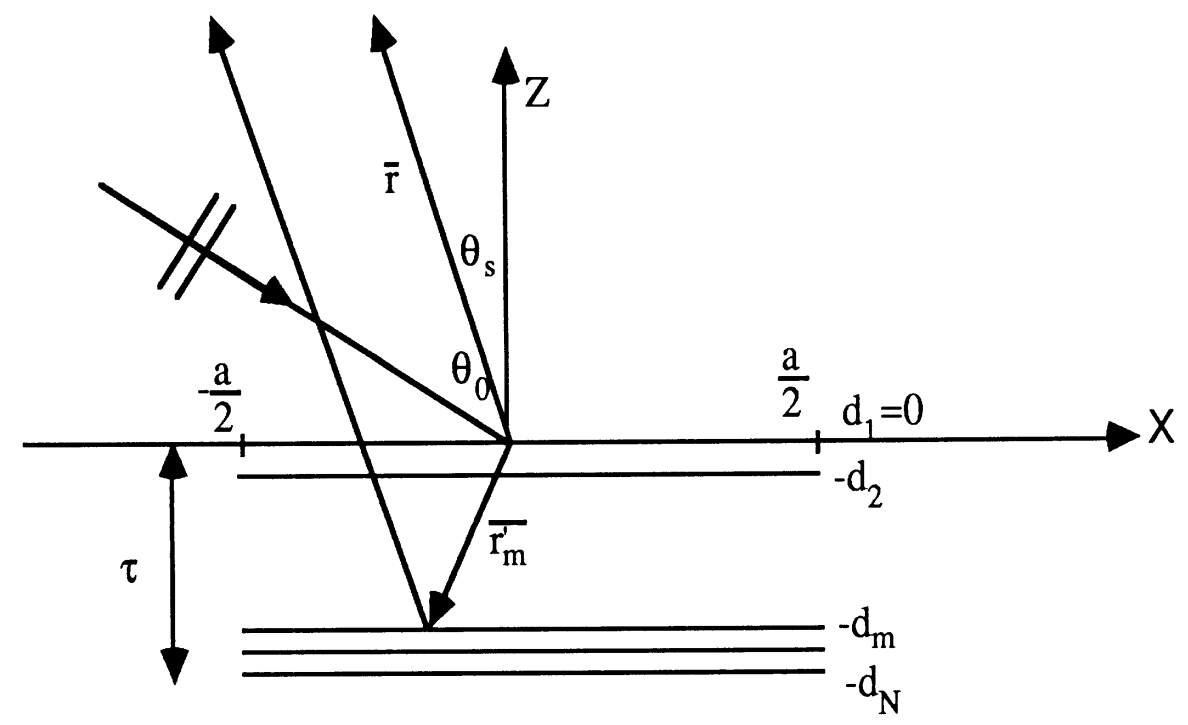

Figure A-2: The geometry of the scattering of a plane wave from a finite N-layer combined-sheet. 